\title{
INTRODUCTION TO GEOGRAPHY OF COVID-19 \\ PANDEMIC: ENVIRONMENTAL ISSUES, PUBLIC HEALTH AND SOCIO-ECONOMIC CONSEQUENCES
}

\author{
Alexander Baklanov ${ }^{1,5,6^{*}}$, Natalia E. Chubarova ${ }^{2}$, Vladimir A. Kolosov³ ${ }^{3}$ Svetlana M. Malkhazova², \\ Boris N. Porfiriev ${ }^{4}$ \\ 'Science and Innovation Department, World Meteorological Organization (WMO), Geneva, Switzerland \\ ${ }^{2}$ Faculty of Geography, Lomonosov Moscow State University, Leninskie Gory 1, Moscow, 119991, Russia \\ ${ }^{3}$ Institute of Geography, Russian Academy of Sciences, Staromonetnyi per., 29, 119017 Moscow, Russia \\ ${ }^{4}$ Institute of Economic Forecasting of the Russian Academy of Sciences, Moscow, Russia \\ ${ }^{5}$ Niels Bohr Institute, University of Copenhagen, Copenhagen, Denmark \\ ${ }^{6}$ Institute of Atmospheric Physics of the Russian Academy of Sciences, Moscow, Russia \\ *Corresponding author: abaklanov@wmo.int \\ Received: November 3' 2021 / Accepted: November 9h 2021 / Published: December $31^{\text {st }}, 2021$ \\ https://doi.org/10.24057/2071-9388-2021-044
}

\begin{abstract}
The COVID-19 pandemic has severely affected all countries and the global scientific agenda, particularly that of health, economy, environment, geography and geosciences in general. This Special Issue is also a contribution to the global efforts of the scientific community in the analysis of the geography of the COVID-19 pandemic with public health, economic and environmental consequences. Two blocks of papers are considered: (1) the socio-spatial, statistical and geographical analysis of COVID-19 distributions; and (2) the impacts of the pandemic lockdown on the environment, air pollution, and the quality of water.
\end{abstract}

KEYWORDS: COVID-19 pandemic, lockdown, environment, air pollution, quality of water, geographical analysis of COVID-19 distribution

CITATION: Alexander Baklanov, Natalia E. Chubarova, Vladimir A. Kolosov, Svetlana M. Malkhazova, Boris N. Porfiriev (2021). Introduction To Geography Of Covid-19 Pandemic: Environmental Issues, Public Health And Socio-Economic Consequences. Geography, Environment, Sustainability, Vol.14, No 4, p. 105-108 https://doi.org/10.24057/2071-9388-2021-044

Conflict of interests: The authors reported no potential conflict of interest.

\section{INTRODUCTION}

The COVID-19 pandemic has greatly affected all countries and the global scientific agenda, particularly that of health, economy, environment, geography and geosciences in general. The pandemic has highlighted the importance of international solidarity and unity in the face of the worst global health and economic crisis in contemporary lifetime. Thousands of papers have been published during the last year in different journals around the world analyzing different aspects of current experience and possible further scenarios.

However, the virus is still with us, with more potent variants. It remains the most immediate challenge for geosciences and health, including its impacts on geoscience development and the achievement of the United Nations Sustainable Development Goals.

Long-term visions based on transdisciplinary scientific advances are therefore essential. As a consequence, the global research community calls for contributions based on data-driven and theory-based approaches to health in the context of global change (Schertzer et al. 2020). This includes: (i) main lessons from lockdowns, (ii) how to get the best scientific results during the corona pandemic? (iii) how to manage field works, geographical monitoring and planetary missions? (iv) qualitative improvements in epidemic modelling, with nonlinear, stochastic, and complex system science approaches; ( $v$ ) eventual interactions between environmental, weather, climate factors and epidemic/health problems; (vi) new surveillance capabilities (including contact tracing), data access, assimilation and multidimensional analysis techniques; (vii) a fundamental revision of our urban systems, their greening and their need for mobility; (viii) a special focus on urban biodiversity, especially to better managing virus vectors; (ix) national and urban resilience must include the resilience to epidemics, and therefore requires revisions of urban governance.

A number of recently published global review papers analyzed experience of different countries in solving the above-mentioned issues.

International symposia and programmes, in particular the World Meteorological Organization, elucidated what is known, understood, and can be reliably predicted about environmental variables' influence on the trajectory of the COVID-19 epidemic, from global, hemispheric, regional and local perspectives. Outcomes of this work (WMO 2021) include a synthesis of the information presented and recommendations for further research at local to global scales. This report presents a summary of key findings to date, as informed by peer reviewed literature. It is motivated both by the global relevance of the subject and by the staggering number of papers and pre-prints currently available, which emphasizes the need for careful review and communication of the state of the science. Main findings include to date: (i) Epidemiological studies of COVID-19 have offered mixed 
results regarding the meteorological sensitivity of the disease; (ii) COVID-19 transmission dynamics appear to have been controlled primarily by government interventions rather than meteorological factors; (iii) Respiratory viral infections frequently exhibit some form of seasonality, however the underlying mechanisms that drive seasonality of respiratory viral infections are not yet well understood; (iv) Laboratory studies have yielded some evidence that the virus survives longer under cold, dry, and low ultraviolet radiation conditions; ( $v$ ) There is evidence that chronic and short-term exposure to air pollution exacerbates symptoms and increases mortality rates for some respiratory diseases. Another global study (Sokhi et al. 2021) provides a comprehensive observational analysis, focuses on changes in air quality in and around cities across the globe for main air pollutants during different phases of the lockdown, with the following conclusions: (i) NO2 - major reductions in emissions (traffic) will be effective but need to be equivalent to full lockdown; (ii) PM - Measures similar to pandemic lockdown will not be effective for decreasing PM2.5 and PM10 to meet WHO guidelines (WHO 2021). Complexities from precursor emissions, chemistry and meteorology; (iii) $\mathrm{O} 3$ - reductions in precursor emissions, chemistry, meteorology and urban background are important; (iv) Updated WHO Air Quality Guidelines pose additional challenges - even during full lockdown there will be guideline exceedances globally; ( $v$ ) International policies need to be more ambitious (e.g. Convention of Long-range Transboundary Air Pollution and the Gothenburg Protocol) (precursor emissions for PM and $\mathrm{O}_{3}$ ); (vi) Complexities from regionally dependent meteorological and episodic emissions from natural or anthropogenic sources (e.g., dust and biomass burning events) can add further complexity; (vii) Potential for co-benefits - reducing the impact of shortlived climate pollutants and GHGs, e.g. cleaner energy transition, low or zero emission traffic, and other control measures targeted at source.

Forster et al (2020) and Jones et al. (2021) analyzed the climate response to emissions reductions due to COVID-19 for current and future global climate scenarios and concluded that (i) Air pollutants interact with climate and have resulted in both warming and cooling over the historical period; (ii) Emission reductions lead to benefits for air quality and health but with differing impacts on climate; (iii) Emission reductions during COVID-19 restrictions impacted air quality but with a small to negligible effect on climate; (iv) Ultimately it is important to consider both climate and air quality policies together to maximize any potential co-benefits in any future measures.

This special issue «Geography of the COVID-19 pandemic: public health, economic and environmental consequences» of the «Geography, Environment, Sustainability» journal is also a contribution to these global efforts to analyze the geography of the COVID-19 pandemic with public health, economic and environmental consequences.

Two main blocks of the papers within the issue can be distinguished: (1) Socio-spatial, statistical and geographical analysis of COVID-19 distributions; and (2) Impacts of the pandemic lockdown on the environment, air pollution, and the quality of water.

\section{SOCIO-SPATIAL AND GEOGRAPHICAL ANALYSIS OF COVID-19}

The problems concerning socio-spatial and geographical analysis of COVID-19 are discussed in the papers (Kolosov et al. 2021; Kieu et al. 2021; Rahardjo et al. 2021; Sahu and Mishra 2021; Vu et al. 2021; Talib 2021; Wetchayont and Waiyasusri 2021).

Kolosov et al. (2021, this issue) reviewed the main areas and methods of human-geographical studies. The authors distinguished between three areas in this field: studies of the spatial spread of infection on the different stages of the pandemic; analysis of its demographic, (geo) political and economic implications, and attempts to forecast the impact of social and technological changes accelerated by the pandemic on territorial structures.

Kieu et al. (2021, this issue) reviewed over hundred scientific papers on applications of GIS and Remote Sensing methods in studies of the COVID-19 pandemic distribution, including spatio-temporal changes, WebGISbased mapping, the correlation between the COVID-19 and natural, socio-economic factors, and the environmental impacts. They provided recommendations on how to apply new techniques to better understand and manage the evolution of the COVID-19 pandemic and effectively assess its impacts.

Rahardjo et al. (2021, this issue) assessed the contribution of crowdsourcing data from social media in understanding locations and geographical distribution patterns of COVID-19 in Indonesia. The accuracy of the resulted data and visualization maps in this study was assessed by comparing the results with the official data from the Ministry of Health of Indonesia. Although the accuracy of crowdsourcing data remains a challenge, the authors argued that crowdsourcing platforms can be a potential data source for an early assessment of the disease spread especially for countries lacking possibilities to build a systematic data structure to monitor the disease spread.

In the paper (Sahu and Mishra 2021, this issue) the Vulnerability Index (VI) distribution over the 28 states and 8 Union Territories of India is discussed. The VI aims at identifying the sources and forms of vulnerability of population due to COVID-19. The results of the assessment support that the factors involved in the three-section exposure, sensitivity, and adaptive capacity had a significant impact on the vulnerability of the population. This study identified that people in Puducherry, Delhi, Goa, Tripura, and Andhra Pradesh are more vulnerable and need more attention from the government and policymakers. The adaptive capacity pattern shows that small states with relatively better medical infrastructure and COVID-19 specific health care facilities can effectively erode the vulnerability. It is expected that the proposed method will have good practical applications.

The analysis of the spatial clustering of the COVID-19 pandemic using spatial auto-correlation analysis is shown in (Vu et al. 2021, this issue) for conditions of the Fourth COVID-19Wave in Vietnam. The spatial clustering including spatial clusters (high-high and low-low), spatial outliers (low-high and high-low), and hotspots of the COVID-19 pandemic were explored using the local Moran's I and Getis-Ord's statistics in two phases of the fourth COVID-19 wave. Significant low-high spatial outliers and hotspots of COVID-19 were first detected in the north-eastern region in the first phase, whereas, high-high clusters and low-high outliers and hotspots were then detected in the southern region of Vietnam. The present findings confirm the effectiveness of spatial autocorrelation in the fight against the COVID-19 pandemic, especially in the study of spatial clustering of COVID-19.

The results presented in (Talib 2021, this issue) also concern the application of geospatial analysis of the data in South-Eastern Asia. The author provides an accurate estimation of the cluster tracking system of the 
COVID-19 by using geospatial technology in Malaysia. This study is important for raising public awareness of the virus, especially among Malaysian citizens making them more concerned, obeying all the Standard Operating Procedure provided by the government to prevent the spread of COVID-19.

The geospatial analysis has also been applied in (Wetchayont and Waiyasusri, 2021, this issue) for the detection and monitoring of the spreading stage during Third Wave of the COVID-19 Pandemic in Thailand. The authors showed that Bangkok, the capital of Thailand, was a significant hotspot for incidence rates, whereas other cities across the region have been less affected. Bivariate Moran's I showed a low relationship between COVID-19 incidences and the number of adults, whereas a strong positive relationship was found between COVID-19 incidences and population density. Daily changes in global Moran's I successfully indicated the Early and Spreading stages during the third wave of the COVID-19 pandemic. These findings could be used to create monitoring tools and aid in policy prevention planning.

\section{IMPACTS OF THE PANDEMIC LOCKDOWN ON THE ENVIRONMENT, AIR POLLUTION, AND THE QUALITY OF WATER}

The impacts of the pandemic lockdown on the environment, air pollution, and water quality are considered in (Chubarova et al. 2021; Sari et al. 2021; Islam et al. 2021; Arakelov et al. 2021). The analysis of changes in concentration of different atmospheric gas and aerosol species during the COVID-19 lockdown has been made using ground-based and satellite measurements for different geographical regions: Iran (Sari et al. 2021), India (Islam et al. 2021) and Moscow megacity in Russia (Chubarova et al. 2021).

In (Chubarova et al. 2021, this issue) the dynamics of the atmospheric pollutants and meteorological conditions has been analyzed during the lockdown due to the COVID-19 pandemic in spring-summer of 2020 in Moscow, Russia, according to the ground-based measurements. The decrease in traffic emissions during the lockdown played an important role in the decrease (up to 70\%) and much smoother daily cycles of many gaseous species and aerosol PM10 concentrations. On contrary, there was an increase in surface ozone concentration (up to 18\%). The additional comparisons of situations with similar synoptic conditions of northern advection, prevailing during the lockdown period, with additional removing the cases affected by fire smoke advection, have revealed similar tendencies, but with the substantial decrease in absolute concentrations, except ozone. The authors obtained a statistically significant negative correlation between the Yandex self-isolation indices (SII), which can be used as an inverse proxy of traffic intensity, and daily concentrations of all pollutants, except surface ozone, using Pearson partial correlation analysis with fixed temperature factor. In situations with $\mathrm{SII}>2.5$ more favorable conditions for surface ozone generation were observed due to smaller $\mathrm{NO} x$ and the higher $\mathrm{O}_{3} / \mathrm{NO}_{x}$ ratios at the same ratio of VOC/NOx. The ozone increase during lockdown may also happen, since in conditions with the prevailing northern air advection the growth of ozone could be observed due to the downward flux of the ozone-rich air from the higher layers of the atmosphere.
In (Sari et al., 2021, this issue) the columnar content of main polluted trace gases $\mathrm{NO}_{2}, \mathrm{CO}$ and $\mathrm{SO}_{2}$ was analyzed over Iran and its several districts with high population. The study was based on the retrievals from the TROPO spheric Monitoring Instrument (TROPOMI) on board ESA Sentinel -5P mission as a part of Copernicus program. The concentrations of the polluted gases observed during the lockdown period were compared with those for the period before and after the COVID-19 lockdown. The authors showed that the content of these gases significantly decreased, especially over the urban areas with high population activity such as Tehran and some other large industrial cities (about $80 \%$ for $\mathrm{SO}_{2}$, up to $50 \%$ for $\mathrm{NO}_{2}$ and (O). Some effects of the decrease in the content of the pollutants were also observed after lockdown period compared with pre-lockdown conditions.

Sentinel-5P TROPOMI data were also used in the analysis of the columnar content of nitrogen dioxide due to the lockdown during the COVID-19 pandemic in India (Islam et al. 2021, this issue). The spatiotemporal characteristics of the tropospheric column $\mathrm{NO}_{2}$ concentration during 45 days of the lockdown were compared with the same days of 2019. Results showed that the mean $\mathrm{NO}_{2}$ concentration reduced from 0.00406 $\mathrm{mol} / \mathrm{m}^{2}$ before the lockdown (2019-03-25 to 201905-10) to $0.0036 \mathrm{~mol} / \mathrm{m}^{2}$ during the lockdown period (2020-03-25 to 2020-05-10). The maximum decline of $\mathrm{NO}_{2}$ concentration was observed in large cities like Gautam Buddha Nagar (- 40.5\%) and Delhi (- 37.5\%). Positive standard residuals in the results of the applied Ordinary Least Squares (OLS) method indicate that the concentration of $\mathrm{NO}_{2}$ has reduced more than expected in the OLS model. The large value of z-score (24.11) from spatial autocorrelation showed that residuals are highly clustered and there is less than a 1\% likelihood that this clustered pattern could be a result of a random chance.

The effects of lockdown due to COVID-19 on the quality of water were studied in Arakelov et al. 2021, this issue). In this paper the results of the hydrochemical analysis of the Kuban's Black Sea Waters were considered in the conditions of the 2020 quarantine. They concluded that its most visible impact was a disproportionate decrease of the recreational pressure which reduced the concentration of the main pollutants. The authors revealed a proportional relationship between biochemical oxygen demand and the concentration of mobile forms of ammonium nitrogen. Interestingly, though the petroleum refineries in the city of Tuapse did not stop during the quarantine period, the concentration of petroleum derivates dropped sharply.

\section{CONCLUSION}

The COVID-19 pandemic is the most immediate challenge for geosciences and health, including its impacts on geoscience development and the achievement of the United Nations Sustainable Development Goals. The papers in this special issue of Geography, Environment, Sustainability would be helpful for understanding the problems in economical, socio-spatial and geographical analysis of COVID-19 pandemic and its consequences, as well as in its impact on the environment, air pollution, and the quality of water. 


\section{REFERENCES}

Arakelov M. S. , Lipilin D. A., Dolgova-Shkhalakhova A. V. (2021). Influence of Quarantine Measures Against COVID-19 on the State of Black Sea Coastal Waters. Geography, Environment, Sustainability, 4(14), 199-204, DOI: 10.24057/2071-9388-2021-089.

Chubarova N. Ye., Androsova E. Ye., Lezina E. A. (2021). The Dynamics of the Atmospheric Pollutants during the Covid-19 Pandemic 2020 and their Relationship with Meteorological Conditions in Moscow. Geography, Environment, Sustainability, 4(14), 168-182, DOl: 10.24057/2071-9388-2021-012.

Forster P.M., Forster H.I., Evans M.J. et al. (2020). Current and future global climate impacts resulting from COVID-19. Nat. Clim. Chang, 10, 913-919, DOI: 10.1038/s41558-020-0883-0.

Jones C.D., Hickman J.E., Rumbold S.T., Walton J., Lamboll R.D., Skeie R.B., et al. (2021). The climate response to emissions reductions due to COVID-19: Initial results from CovidMIP. Geophysical Research Letters, 48, e2020GL091883, DOI: 10.1029/2020GL091883.

Kolosov V.A., Tikunov V.S., Eremchenko E.N. (2021). Areas of Socio-Geographical Study of the COVID-19 Pandemic in Russia and the World. Geography, Environment, Sustainability, 4(14), 109-116, DOI: 10.24057/2071-9388-2021-091.

Rahardjo Noorhadi, Djarot Heru Santosa, Hero Marhaento (2021). Crowdsourcing Data to Visualize Potential Hotspots for Covid-19 Active Cases in Indonesia. Geography, Environment, Sustainability, 4(14), 125-130, DOl: 10.24057/2071-9388-2021-011.

Sahu Netrananda, Martand Mani Mishra (2021). Assessing the Vulnerability Index of COVID-19 Pandemic in India. Geography, Environment, Sustainability, 4(14), 131-139, DOI: 10.24057/2071-9388-2021-059.

Sari Nurwita Mustika, Muhammad Nur Sidiq Kuncoro (2021). Monitoring of CO, $\mathrm{NO}_{2}$ and $\mathrm{SO}_{2}$ Levels During the COVID-19 Pandemic in Iran Using Remote Sensing Imagery. Geography, Environment, Sustainability, 4(14), 183-191, DOI: 10.24057/2071-9388-2020-74.

Schertzer D. et al. (2020). Great e-Debate «Epidemics, Urban Systems and Geosciences». «EGU2020: Sharing Geoscience Online». European Geosciences Union (EGU) session ITS2.10 / NP3.3: «Urban Geoscience Complexity: Transdisciplinarity for the Urban Transition».

Sokhi R.S., V Singh X Querol, Finardi S., Créso Targino A., de Fatima Andrade M., Pavlovic R., Garland R.M., Massagué J., Kong S., Baklanov A., Ren L., Tarasova O., Carmichael G., Peuch V-H., et al., (2021). A global observational analysis to understand changes in air quality during exceptionally low anthropogenic emission conditions, Environment International, 157, 106818, DOI: 10.1016/j.envint.2021.106818.

Talib Noorfatekah, Nur Nabila Mohd Fuad, Nurhafiza Md Saad, Nurul Ain Mohd Zaki, Nurhanisah Hashim (2021). Towards a Strategic Approach of COVID-19 Cluster Web Mapping in Malaysia. Geography, Environment, Sustainability, 4(14), 148-154, DOI: 10.24057/2071-93882021-088.

Vu Danh-tuyen, Tien-thanh Nguyen, Anh-huy Hoang (2021). Spatial Clustering Analysis of the COVID-19 Pandemic: A Case Study of the Fourth Wave in Vietnam. Geography, Environment, Sustainability, 4(14), 140-147, DOI: 10.24057/2071-9388-2021-086.

Wetchayont Parichat, Katawut Waiyasusri (2021). Detection and Monitoring of the Spreading Stage during Third Wave of the COVID-19 Pandemic in Thailand Using Moran's I Approach. Geography, Environment, Sustainability, 4(14), 155-167, DOI: 10.24057/2071-9388-2021-090.

WHO (2021). WHO global air quality guidelines. Particulate matter (PM2.5 and PM10), ozone, nitrogen dioxide, sulfur dioxide and carbon monoxide. Geneva: World Health Organization; Licence: CC BY-NC-SA 3.0 IGO.

WMO (2021). First Report of the WMO COVID-19 Task Team: Review on Meteorological and Air Quality Factors Affecting the COVID-19 Pandemic (WMO-No. 1262). Part I: First COVID-19 report, Part II: Executive Summary (multilingual). World Meteorological Organization, https://library.wmo.int/index.php?lvl=notice_display\&id=21857\#.YZF8GGDMI2w 